\title{
Condicionantes biosociales de las enfermedades no diagnosticadas ${ }^{1}$
}

\section{Biosocial Conditionants of the Undiagnosed Diseases}

\author{
Juan R. Coca ${ }^{2}$ \\ Universidad de Valladolid (España) \\ ORCID: https://orcid.org/0000-0003-1140-7351
}

\author{
Juan Antonio Roche Cárcel ${ }^{3}$ \\ Universidad de Alicante (España) \\ ORCID: https://orcid.org/0000-0003-1522-5918
}

\section{Juan Antonio Rodríguez Sánchez ${ }^{4}$ \\ Universidad de Salamanca (España) \\ ORCID: https://orcid.org/0000-0002-9949-9168}

Recibido: 04-11-2020

Aceptado: 24-12-2020

\footnotetext{
${ }^{1}$ El presente trabajo de investigación se realiza en el marco del proyecto "Investigación, redes asistenciales y empoderamiento: respuestas sociales y científicas a las enfermedades raras en la Península Ibérica (1940-2015)" HAR2017-87318-P (Programa de I+D Excelencia), financiado por el Ministerio de Economía, Industria y Competitividad y dirigido por el profesor Rodríguez Sánchez.

2 (juancoca@soc.uva.es) Profesor contratado doctor del Dpto. de Sociología y Trabajo Social de la Universidad de Valladolid. Coordinador de la Unidad de Investigación social en salud y enfermedades raras. Ha publicado más de un centenar de artículos y libros tales como La comprensión de la tecnociencia.

${ }^{3}$ (ja.roche@ua.es) Profesor titular del Dpto. Sociología I de la Universidad de Alicante. Ha publicado numerosos artículos, capítulos y libros. Dentro de los libros más recientes está Entre el Monte de Apolo y la vid de Dioniso. Naturaleza, Dioses y Sociedad en la arquitectura teatral de la Grecia Antigua.

${ }^{4}$ (jarshm@usal.es) Profesor titular del Área de Historia de la Ciencia de la Universidad de Salamanca. Sus investigaciones se han centrado en los imprecisos límites entre las prácticas médicas oficiales y las consideradas alternativas. Desde 1997 comenzó a trabajar en la historia de la poliomielitis y el síndrome postpolio y desde 2005 es investigador principal del proyecto "Estudio comparativo de los cambios sociales y profesionales relacionados con la polio en el área transfronteriza hispanoportuguesa desde 1956", financiado por el Ministerio de Educación.
} 


\title{
Resumen
}

En este trabajo de investigación nos aproximamos al conocimiento de las enfermedades no diagnosticadas. Dichas enfermedades se enmarcan dentro de las llamadas enfermedades raras, debido a que no son frecuentes. Generan una serie de condicionantes en la vida de las personas afectadas y en sus relaciones sociales. Ello es fruto de la ruptura semiótica entre el código biológico y el social. Esta situación puede obligar a las personas con estas enfermedades a estar situadas fuera de la estructura social y ver reducido su capacidad para tomar decisiones. Por esta razón es necesario desarrollar puentes semióticos que conecten los códigos de los que hemos hablado.

Palabras-clave: Normalidad, estructura social, normalidad, diversidad, biosemiótica.

\begin{abstract}
In this research work we approach to the knowledge of undiagnosed diseases. These diseases are framed within the so-called rare diseases, because they are not frequent. They generate a series of conditions in the lives of the affected people and in their social relationships. This is the result of the semiotic break between the biological and the social code. This situation can force people with these diseases to be out of the social structure, and they also can be reduced their capacity to make decisions. For this reason, it is necessary to develop semiotics bridges that connect the codes we have talked about.
\end{abstract}

Key-words: Normality, Social Structure, Normality, Diversity, Biosemiotic.

\section{Introducción}

Las enfermedades no diagnosticadas plantean, a nuestro juicio, uno de los mayores retos epistemológicos en la actualidad, en relación con los factores pragmáticos que intervienen en la construcción, estructuración y operatividad de la ciencia (Moulines, 2011). Estas enfermedades son un conjunto de patologías poco frecuentes, de las que o bien todavía no existe conocimiento suficiente para determinar biomédicamente qué es lo que le sucede a una persona, o bien los enfermos se ven afectados por el desconocimiento médico. Si bien se considera que las personas que se encuentran en esta situación son un $6 \%$ del conjunto de las que constituyen el conjunto de quienes padecen enfermedades "raras" (Tifft y Adams, 2014). Este desconocimiento implica que 
no existen mecanismos experimentales que permitan aproximar a la comunidad científica a la detección y designación rápida de las patologías o de los factores causales de tales afecciones.

Tal y como veremos posteriormente, podemos afirmar que estas enfermedades generan una "ruptura" en el código semiótico entre lo biológico (los elementos causales) y los sociales (la propia enfermedad). De ahí que la mayor parte de los esfuerzos científicos (biomédicos, biológicos, etc.) estén centrados en la identificación y construcción de este código semiótico. En este punto es, precisamente, donde se encuentra el reto epistemológico que, además, implica un ineludible condicionante diagnóstico.

EURORDIS, la alianza no gubernamental de organizaciones de pacientes, diferencia en su página web entre enfermedades que todavía no tienen diagnóstico y las enfermedades sin diagnóstico que, habitualmente, se conocen como "Síndromes sin nombre" (SWAN, según su acrónimo inglés: Syndrome without a name). Las primeras (las que todavía no tienen diagnóstico) hacen referencia al periplo diagnóstico al que se ven sometidos los pacientes afectados por enfermedades poco prevalentes o raras. Es decir, que en numerosas ocasiones los pacientes se encuentran con modelos de diagnosis biomédica que van desde lo más frecuente a lo menos. Ello hace que se tarde mucho tiempo en descartar patologías más habituales $\mathrm{y}$, por tanto, los pacientes afectados tienden a tardar muchos años en obtener un diagnóstico certero. Así, en este fenómeno social y biomédico la patología es conocida y, por ende, es posible un diagnóstico efectivo.

Las enfermedades sin diagnóstico (sensu stricto), implican un problema relacionado con la ignorancia o con el desconocimiento biomédico. Dicho de otro modo, se desconocen los mecanismos causales de este proceso. Por esta razón, no existe la posibilidad de tener diagnóstico, puesto que el agente causal permanece desconocido. Este es el caso en el que nos centraremos en este trabajo.

En las enfermedades no diagnosticadas el paciente se ve afectado por los elementos subjetivos de los factores pragmáticos que conforman la Ciencia. De tal manera que, en función de la expertise del médico o de la intencionalidad del mismo, el paciente podrá estar más arropado o menos por el sistema biomédico. Ortega et al (2012) están en línea con lo que estamos exponiendo, cuando indican que el proceso de diagnosis puede estar afectado negativamente por pensar en lo más prevalente, tal y como, según estos autores, expuso J. Skoda a finales del siglo XIX. Por ello, estos autores apuestan por la combinación entre el rigor de la medicina basada en la evidencia y el diagnóstico intuitivo. Pese al interés de este enfoque tan prudente, mantiene el problema básico de las enfermedades no diagnosticadas, que -como su nombre indica- no tienen diagnóstico, por lo que el elemento intuitivo se incrementa (con el consiguiente 
problema de subjetividad), mientras que el componente más objetivo (las evidencias biomédicas) se reduce.

Dado el conocimiento existente sobre las enfermedades raras (también llamadas enfermedades minoritarias, de baja prevalencia o poco frecuentes), la comunidad científica biomédica es consciente de su existencia y de que las personas que las sufren están realmente enfermas. Ahora bien, existen diversos determinantes biológicos, psicológicos, sociales, económicos, imaginarios, etc. que influyen en la vida de las personas afectadas por estas patologías. En este sentido es necesario advertir que no podemos ocuparnos de todos los condicionantes que afectan a este grupo de individuos. Por esta razón, centraremos nuestro interés en los factores que afectan a la estructuración social de este grupo y, para ello, usaremos las propuestas de Margaret S. Archer en diálogo con los estudios biosociales sobre estas enfermedades.

\section{Estructuración social anormativa}

Las investigaciones teóricas de los últimos tiempos sobre la ontología social de corte estructural han ampliado sustancialmente el conocimiento de los fenómenos que dan forma al sistema social. Resulta esclarecedora la distinción que realiza Raimo Tuomela (2010 y 2013) entre el modo-yo (I-Mode) y el modo-nosotros (We-Mode) para designar los diferentes modos en los que opera la intencionalidad dentro del ámbito social. Supongamos que una persona $A$ tiene una intención $i$ para el desarrollo de su acción. Esta intencionalidad puede variar, y de hecho así lo hace, en función de si la decisión es individual o está mediada por un grupo social. Por esta razón, este autor considera que las personas tenemos dos modos diferentes de estar en el mundo: como sujetos individuales y como sujetos colectivos. Al respecto, Tuomela (2013) indica que la intencionalidad hace referencia a los diferentes estados mentales de las personas, a saber: creencias, deseos e intenciones.

Estos estados mentales se verán modificados en función del modo, individual o colectivo, a través del cual nos enfrentemos a la realidad. Dichos estados estarán estrechamente relacionadas con las emociones y los sentimientos, e incluso -añadimos nosotros- con los imaginarios sociales (Coca, 2017). Individualidad y socialidad son modos diferentes y análogos de estar vertidos a la realidad (Zubiri, 1998) y, además, presentan cierto nivel de continuidad en los diferentes organismos (Coca et al, 2020).

Raimo Tuomela (2010 y 2013), en línea con Habermas (2018) plantea un enfoque racional de la socialidad y de la socialización. No obstante, no entra a considerar las famosas consecuencias no intencionadas de la acción social. Aunque son numerosos los sociólogos que se han ocupado de las consecuencias 
no intencionales o no deseadas de la acción -E. Lamo de Espinosa (1990), R. Ramos (1993), N. Luhmann y De Giorgi (1993), N. Elias (2000) y A. Giddens (2013), entre otros-, es necesario recordar que fue Robert Merton (1936) quien hace muchos años comenzó con el estudio de estos aspectos derivados de la acción social. Merton, desde una perspectiva claramente racionalista, identifica cuatro elementos que traen consigo tales consecuencias no previstas: la ignorancia, el error, la inmediatez de los intereses de las personas y las predicciones contraproducentes. La articulación mertoniana de estas consecuencias no intencionadas son claramente negativas, pero resulta lógico pensar que estos resultados emergentes de la acción social también pueden ser positivos. Por ello, Boudon (1982) mostró en su estudio sobre los tipos de consecuencias no intencionadas que éstas podrían generar resultados beneficiosos, problemas o ambos. Dichos resultados pueden provenir, a su vez, del hecho de que nadie consiga sus objetivos, de que algunas personas logren sus intereses o de que todos los consigan. En esta breve aproximación a las consecuencias no intencionadas de la acción, no podemos olvidarnos de Giddens (2013), quien mostró, entre otras cosas, que están estrechamente relacionadas con la morfogénesis social y con los elementos normativos del sistema social. Por todo ello, podemos afirmar que la estructura ontológica social no es exclusivamente racional, sino también arracional.

Las personas se organizan en grupos sociales, los cuales, a su vez, operan en base a procesos de interacción internos y externos. Estos grupos sociales, además, desarrollan acciones comunes y median en la estructuración de la sociedad. Ahora bien, los diferentes grupos de un sistema social no son reducibles (conceptualmente) a las propiedades y relaciones establecidas entre los miembros del mismo (Tuomela, 2013). Tanto es así que diferentes grupos pueden desarrollar acciones sociales semejantes e incluso distintas personas pueden pertenecer a grupos diferentes. Ahora bien, ello no quiere decir que no sea posible establecer ciertas tipologías sociales que permitan seguir avanzando en el estudio de los condicionantes sociales. En este sentido podemos indicar que el concepto de sociotipo presenta unas virtudes epistemológicas y metodológicas que no es posible obviar. Además, este concepto permite utilizar con solvencia (ya que ha sido ampliamente estudiado) la concepción ecológica de las enfermedades raras que defiende Francesc Palau (2020).

Antes de continuar es necesario aclarar que el concepto de sociotipo no es un término cerrado e inmutable. Al contrario, este tipo ideal es un modelo explicativo que evoluciona en interacción con el medio ambiente y con los condicionantes internos de las personas incluidas en él. Lo interesante del sociotipo es que tiene la virtud epistemológica de permitir el análisis de un grupo social con unas posibilidades heurísticas que no presentan otros conceptos. En este sentido, podría pensarse que no es necesario hablar de sociotipo y hacer 
referencia al grupo de manera genérica. El problema que podríamos encontrar, entonces, es que perdamos rigor analítico (algo fundamental en los trabajos de investigación). Por otro lado, también debemos indicar que somos conscientes de que este concepto tiene dificultades en la investigación social de carácter general (macro) y cuya base de diferenciación sea la propia sociedad. Sin embargo, permite considerar que los tipos sociales se estructuran a partir de determinadas relaciones humanas (intersubjetivas) y a interacciones entre los individuos de dicho grupo con su ambiente (Marijuán et al, 2017). Dicho de otro modo, un tipo de comportamiento social, o la reunión de personas según una determinada acción o intención, no permite establecer un sociotipo, aunque sí posibilita hablar de la existencia de un grupo social.

Restringiremos el concepto de sociotipo a aquellos individuos que, por factores biológicos, psicológicos y sociales duraderos en el tiempo, presenten condicionantes, determinantes y comportamientos más o menos estables. De este modo, el concepto de sociotipo, según Berry (2011), presenta ciertas analogías con los de genotipo y fenotipo, y es concebido como un término integrador que abarca factores internos y externos y que supone la conjunción de aspectos biológicos, psicológicos y sociales. Por otro lado, los sociotipos permiten estudiar de manera acotada la estructuración y la morfogénesis del sistema social. En este sentido, podemos percatarnos que, desde este enfoque analítico, es posible también acotar la detección de las consecuencias no intencionadas de la acción social relacionada con un determinado sociotipo.

Al fin y al cabo, una visión estratificada y realista de las personas permite diferenciar las propiedades de los individuos en función del nivel estructural en el que se encuentran dentro del sistema social. Asimismo, las personas presentan diferentes capacidades para ejercer su poder (causal) dentro del sistema en base a sus interrelaciones y a las capacidades de agencia social (Archer, 2009). Vemos, entonces, que existe una interfaz entre la estructura social y la agencia en la que, además, se generan procesos emergentes que transcienden los elementos del sistema. De hecho, el concepto de agencia en Archer (2010) implica la interrelación entre la estructura del sistema social, la cultura y, por supuesto, las personas, generando un proceso de agencia social que no puede ser determinado previamente por la estructura.

La agencia y la estructura están, por tanto, interpenetradas. Ahora bien, la perspectiva estructuralista de Archer podría ser considerada como un tanto excluyente de otras alternativas de investigación posibles tales como, por ejemplo, la sociohermenéutica. Por esta razón, consideramos que es necesario plantear un enfoque de análisis trans-subjetivo (Ginev, 2016) donde sea compatible analizar elementos que incluyan a la subjetividad, a la intersubjetividad y a otros aspectos biosociales (las relaciones entre el sistema social y el sistema natural). Esta trans-subjetividad, a nuestro juicio, enlaza 
adecuadamente con la concepción del nicho social donde la sociedad se va paulatinamente estructurando desde los determinantes y los condicionantes del nicho ecológico donde la propia sociedad se encuentra. Decimos esto, puesto que Dimitri Ginev (2016) afirma que la trans-subjetividad es precisamente prenormativa y está vinculada con el modo de ser en el mundo del que tantas veces han hecho mención los hermeneutas (Heidegger, 1971; Gadamer, 1984; Ricoeur, 1996; Grondin, 2003). Por todo esto, el planteamiento epistemológico asentado en la phronesis defendido por Bent Flyvbjerg (2001) tiene una virtud que parece no ser contemplada por Archer. Y es que, para este último autor, la investigación centrada en la phronesis focaliza su atención tanto en el actor (sujeto agente) y en la propia estructura. De ahí que esta perspectiva de análisis transcienda el dualismo entre el actor y la estructura, entre la hermenéutica y el estructuralismo, así como entre el voluntarismo y el determinismo. La agencia será analizada en relación con la estructura, y viceversa. Ahora bien, siendo conscientes (afirmamos nosotros) que existen procesos emergentes que no pueden ser dejados a un lado. Por esta razón, son muy acertadas las afirmaciones de Reed (2011) cuando indican que los significados intersectan entre dos realidades causales generando, a través de sus fuerzas, una forma determinada a lo social. Ello implica que la interpretación de los signos sociales (sociosemiótica) será central para poder, así, construir la explicación causal. Ahora bien, como hemos dicho antes, también existen unos condicionantes trans-subjetivos (vinculados al nicho ecológico y a los aspectos ontológicos de la humanidad) que conducen nuestra argumentación al prisma semiótico constituido por la biosemiótica y la sociosemiótica.

Margaret S. Archer (2016) ha indicado recientemente que la morfogénesis social se ha intensificado en la Modernidad tardía, transformando la estratificación del sistema, alterando el trabajo, las instituciones, los mecanismos políticos y, además, afectando de manera negativa a los procesos de integración social. Con anterioridad a esta época, la morfostasis era más predominante, lo cual -de un modo más difuso- concuerda con la duplicidad entre las metáforas de la modernidad sólida y la modernidad líquida expuesta por Zygmunt Bauman (2003) en su conocida Modernidad líquida. Roche (2009) interpreta este incremento de la morfogénesis social como una desestructuración de la visión tradicional y ancestral basada en la búsqueda del orden y, por tanto, de la morfostasis. Desestructuración relacionada con la desrealidad o con la desmaterialización y con la transformación del tiempo. Así es, ahora se exalta el presente, que es contraído y convertido en omnipresente y tirano (Beriain, 2008), así como lo fluido, devenido en la tardomodernidad todavía más volátil, inmaterial, ingrávido y aéreo que en la modernidad (Sánchez Capdequí, 2004). También se pone el acento en el cambio generalizado, convertido en una forma de vida (Farson, 1973), en un imperativo y en un fin en sí mismo 
(Bauman, 2004). A ello se le suma una dominante discontinuidad espacio temporal (Koselleck, 2016) y la conciencia de la acelerada evanescencia temporal -Ch. Baudelaire (2004); W. Benjamin (Picó, 1988)-, así como la prevalencia del acontecimiento entendido como figura del desorden (Balandier, 2014) y el triunfo del devenir (Baumer, 1985). Con él, se produce lo contingente (Luhmann, 1996b), lo incierto y lo arriesgado (González, 2006), lo fugaz, lo transitorio, lo elusivo, lo efímero (Thiebaut, 1996; Shattuck, 1991), lo instantáneo (Bauman, 2003) -eternizado (Maffesoli, 2000)-, lo inestable, lo disperso, lo rupturista, lo inesperado, lo inédito, lo accidental, lo incontrolado, lo precario, lo imprevisible, lo aleatorio, lo desconocido y lo novedoso: "La modernidad es el movimiento más la incertidumbre" (Balandier, 2014).

En esta evanescencia espacio-temporal, el nicho social también está siendo alterado, con las consiguientes consecuencias biosociales no intencionadas (un ejemplo muy oportuno lo encontramos actualmente en la pandemia causada por el SARS Cov-2). De ahí que los procesos de normativización o anormativización también se estén transformando con mayor rapidez que antes.

\section{Condicionantes biosociales de las enfermedades raras y no diagnosticadas}

Las enfermedades no diagnosticadas son la expresión de un hueco en el conocimiento. Una brecha en la comprensión de los signos biosemióticos causantes de tales patologías, produciendo una configuración sociosemiótica particular. Dicho de otro modo, las personas con enfermedades raras no diagnosticadas se encuentran, en cierto modo descodificadas y son, por tanto, más evanescentes dentro de las sociedades actuales. No tienen las posibilidades de comunicación que sí poseen otras personas para mostrar a los demás cuál es la significación de su manera de estar en el mundo. Su mundo de la vida (Schutz, 1967) está, en cierto modo, socialmente incomunicado, debido a la brecha de ignorancia a la que se ven sometidas, lo que limita las posibilidades sociales de las personas afectadas. Aclaremos esto un poco más.

Lester F. Ward (1905 y 1921), a comienzos del siglo XX, planteó una concepción organicista de la estructura social que tiene interés por su capacidad heurística y que hoy tiene sus continuadores (Elías, 2000; Veblen 2008; Bellah 2017; Jaspers 2017). Ward mostró una visión analógica entre el conocimiento biológico y el sociológico de la época. Consideraba que era necesario hablar de cuerpos sociales (grupos sociales) para que pudiera producirse el fenómeno de reproducción social y, por lo tanto, de evolución social. Los distintos cuerpos sociales estarían condicionados por diferentes elementos presentes en la estructura social (producción, poder, etc.) y la interacción que se genera entre ellos da lugar a las fuerzas sociogénicas. Estas fuerzas las divide Ward 
(1921) en morales, estéticas e intelectuales. Si recordamos lo que hemos visto sobre la morfogénesis expuesta por Margaret Archer, vemos que existen claras concomitancias entre ambos puesto que la sociogénesis es un correlato de la morfogénesis social.

Pues bien, según Ward existe una analogía entre el organismo de un ser vivo y la sociedad, de tal modo que podemos diferenciar los niveles estructurales sociales, en función de las capas germinales de un organismo. Tenemos, por tanto: el ectodermo, la capa externa y visible que se relaciona biológicamente con el epitelio (de manera genérica) y con el cerebro, lo que supone que esta capa está vinculada al ámbito de la relación; el mesodermo que, en líneas generales, da lugar a los músculos y al tejido conectivo, es decir, las capas intermedias del organismo y, por lo tanto, del cuerpo social; y el endodermo que sería la parte interna del cuerpo (intestino y órganos internos). Aunque las ideas de Ward son un tanto antiguas y no han tenido una gran repercusión, presentan interés histórico por la correlación entre el organismo y la estructura social. De tal manera que, según este autor, podemos comprender mejor la configuración del sistema social desde esta perspectiva orgánica, asimismo también podemos entender y describir los efectos negativos de los determinantes estructurales en el propio sistema. Para Ward los tejidos generados por el ectodermo social son los que tienen la capacidad de relacionar a la sociedad con otras, al tiempo que rigen los designios del cuerpo social. En cambio, los órganos más internos tendrían menos poder, aunque tienen la capacidad de nutrir a todo el organismo social.

En la actualidad se ha incrementado la diversidad social y la forma que ella presenta. El centro de la estructura está ocupado por los grupos o entidades sociales con mayor poder, mientras que en los márgenes o en las zonas internas (según la idea de Ward) estarán los grupos con menor poder o capacidad de decisión. El sociotipo de los afectados por enfermedades no diagnosticadas (lo llamaremos ND) estaría situado en la parte más interna de la sociedad. Dicho de otro modo, el estudio de la realidad de este sociotipo nos permitirá comprender un conjunto de mecanismos y procesos sociales que van a favorecer la evolución de todo el sistema social. Además, si son tenidos en cuenta, también facilitará el establecimiento de procesos instituidos de progreso humano y social. Un ejemplo claro de lo que acabamos de indicar lo han detectado Sabatello et al, (2020) y Kuper et al, (2020) cuando afirman que las respuestas ante la pandemia generada por el SARS Cov-2 deben estar sujetas a normas jurídicas, a los principios básicos de justicia distributiva y a las normas de protección de los grupos más vulnerables.

El hecho de no poder nombrar su realidad biomédica determina la capacidad que los demás (las personas no pertenecientes a dicho sociotipo) tienen para comprenderla. De ahí que se generen una buena cantidad de consecuencias 
no intencionadas fruto de las acciones sociales de ND. Recordemos que las personas nacen en un mundo histórico y social (Reichertz, 1988; Elías, 2000: 14-83; Endress, 2014; Balandier, 2014: 25) que hace que tendamos a ver el mundo de una manera más estática (morfostasis). Ello trae consigo que, cuando nos topamos con personas o grupos sociales que rompen esta estabilidad, nuestra interpretación tienda a converger con esta estabilidad o con aquello que sabemos que es así. Por esta razón, es relativamente habitual que las personas del sociotipo ND tengan problemas de incomprensión e, incluso, de rechazo (Coca, 2019a).

Con el objetivo de generar un mayor conocimiento de los factores causales de las diferentes patologías ND, se están produciendo esfuerzos en el ámbito biomédico para detectar las alteraciones genéticas que permitan identificarlos. Hablamos de genética debido a que la mayor parte de las enfermedades poco frecuentes (donde están las ND) son genéticas. De hecho, se calcula que, de las 6.000-8.000 enfermedades raras (von del Lippe, 2017), alrededor del 72\% son genéticas (Palau, 2020). Por esta razón, se han creado redes internacionales para facilitar la localización de las alteraciones genéticas que permitan diagnosticar más rápidamente a las personas, así como identificar nuevas patologías hasta ahora desconocidas (Taruscio et al, 2015). A su vez, por sus implicaciones éticas, han supuesto la necesidad de integrar a los pacientes en su desarrollo, algo que podría contribuir a un empoderamiento de éstos (Gainotti et al, 2018).

La obtención del diagnóstico permite nombrar y significar socialmente el problema, lo que facilita la relación social. Ello es debido a los procesos de significación inherentes a la dimensión relacional humana. Pensemos, por un momento, en la película Joker. En ella el protagonista se reía de una manera incontrolada provocando una reacción negativa en las personas de su alrededor. Para que los demás pudieran comprender que tenía una afección que se manifestaba de este modo, el personaje presentaba una pequeña tarjeta donde explicaba en una frase su condicionante biosocial. Supongamos ahora que una persona no puede tener la seguridad de que tiene una patología, aunque sabe que está enferma de algún modo. Supongamos también que esta afección le obliga a desarrollar -en línea con el trabajo de Goffman (2009)- una performance que es percibida negativamente por su entorno. Como hemos indicado antes, la proyección pública que hacemos de nosotros mismos (la fachada o la imagen) tiende a institucionalizarse de un modo u otro (morfostasis) y a estabilizar su significación a través de este proceso de institucionalización. Así, una persona en una profesora, un varón, un cliente, etc.

En el caso que estamos indicando $-y$ a causa de la baja frecuencia de las patologías- la mayor parte de las personas no están acostumbradas a las manifestaciones externas (sean las que fueren), produciéndose con frecuencia fenómenos de rechazo social. Si la persona tiene mecanismos de presentación 
de su realidad ante los demás, esta situación puede minimizarse. En cambio, si estos mecanismos son inexistentes, cabe la posibilidad de una creciente incomprensión (que puede ser intencionada o no) e, incluso, una exclusión social. Esto último es un fenómeno frecuente en el ámbito de las enfermedades raras (Coca, 2019b). Estas consideraciones, obviamente, son de carácter social. Ahora bien, disciplinas como la nosología y la nosotaxia han desarrollado modos de dar nombres a lo que el paciente vive. Aunque estos procesos tienen un carácter fundamentalmente descriptivo. Por ejemplo, la palabra idopático acompaña a algunos diagnósticos que se componen de términos descriptivos (o de familia de enfermedades) que indican que se desconocen sus causas.

Por otro lado, no es conveniente obviar el impacto negativo que genera saber que uno tiene una enfermedad rara o que tu hijo la posee. La sensación de incertidumbre se incrementa notablemente (Coca, 2019b). Una de las razones es, precisamente, el gran desconocimiento existente y la posibilidad de que no exista tratamiento o fármaco que pueda mejorar la vida de los afectados. Se construye, entonces, una interpretación de la enfermedad donde el miedo, la tragedia y la ausencia de futuro tienen un lugar destacado (Coca et al, 2019)

Sabemos que la mayor parte de las enfermedades raras tiene una causa de origen genético. Además, también conocemos que siguen, en su mayor parte, un patrón hereditario mendeliano (Berman, 2015). Este tipo de patrón hereditario implica que los genes causantes de la enfermedad se heredan de uno o de los dos progenitores, de tres maneras posibles:

i. siguiendo un tipo de herencia autosómica dominante.

ii. a través de un mecanismo autosómico recesivo.

iii. ligado a los cromosomas sexuales (el X o el Y).

Al respecto, recordemos que los genes eucariotas están formados por dos tipos de elementos, los codificantes que reciben el nombre de exones, y los no codificantes que se llaman intrones. Los genes causantes de enfermedades minoritarias estarán, por tanto, en las regiones codificantes (llamado genéricamente exoma) y es allí donde los genetistas intentan detectar las nuevas enfermedades. Volveremos sobre esto.

Por otro lado, es bien conocido que los organismos eucariotas tienen en sus células dos copias del material genético de sus ascendientes (una de origen paterno y, la otra, materna). En ese proceso de reparto del material genético, los descendientes pueden recibir una copia de los genes causantes de la enfermedad. Si la herencia es autosómica dominante, las personas que manifiestan la patología lo hacen al tener una copia de un progenitor portador. No obstante, 
también debemos ser conscientes de que es posible que suceda una alteración genética de novo. Si los progenitores no manifiestan la patología, entonces podemos estar ante una herencia recesiva. Una persona con una alteración genética recesiva y nueva, no manifestará la enfermedad (precisamente por ese carácter recesivo), aunque lo podrá transmitir a su descendencia. Por último, también puede darse un fenómeno de herencia ligada a los cromosomas $\mathrm{X}$ e Y. En ella nos encontramos que, o bien los varones (si la afección está relacionada con el cromosoma Y), o bien las mujeres (si la enfermedad se manifiesta cuando dos cromosomas $\mathrm{X}$ están alterados), estarán afectados por la enfermedad. Es habitual, en estos casos, comprobar que en una de las dos familias de los progenitores (la del padre o la de la madre) existen casos en personas del mismo sexo. Ello ayuda a la detección de algunas enfermedades.

Berman (2015) advierte que las enfermedades monogénicas, aunque están causadas por la alteración de un único gen, no son tan sencillas como se pudiera pensar a priori. En este sentido, conviene tener presente que el $\mathrm{ADN}$, nuestro material genético, es transcrito a ARN y que éste codifica las proteínas. Por lo tanto, cabe pensar que, si conocemos el gen defectuoso, podremos identificar la proteína alterada, comprender la patología y generar mecanismos que solucionen el problema en cuestión. Ahora bien, la realidad es mucho más compleja. Berman (2015) indica que una proteína monogénica puede variar de función dependiendo del lugar específico del organismo y del tipo de mutación del gen. Por ejemplo, la alteración del gen CEP290 genera diferentes patologías tales como el Síndrome de Bardet-Biedl, el Síndrome de Joubert, la Amaurosis congénita de Leber, el Síndrome de Meckel y el Síndrome de Senior-Loken (Berman, 2015). Además de esta variación en la función proteica, también puede darse el caso de que un mismo gen codifique una determinada proteína que afecta, a su vez, a otras y ocasionar patologías en diferentes tejidos del organismo. Por último, una misma proteína puede cumplir funciones diferentes en distintas células del organismo y también puede variar su función dependiendo del estado de desarrollo del organismo. Estos ejemplos que acabamos de mencionar permiten comprender como es posible que la alteración de un solo gen provoque síndromes (cuadro patológico múltiple).

Además de lo que hemos indicado, existen todavía más determinantes biológicos, pero creemos que, con lo que expuesto, queda clara la argumentación. La alteración de un gen puede afectar a las vías moleculares relacionadas y generar patologías. Ahora bien, sería simplista afirmar que un solo gen puede producir una determinada patología por sí mismo. Es necesario que estos cambios traigan consigo otros efectos posteriores negativos. Esto es, la modificación de un gen debe traer consigo la alteración de las vías subsiguientes para que realmente pueda llegar a ser un factor causal de una patología. 
Las enfermedades poco frecuentes (raras) pueden generar una serie de alteraciones en el fenotipo de las personas afectadas. No obstante, no pensemos que la correlación entre la variación de un gen y un determinado comportamiento social implica que en cualquier nicho social posible (presente o futuro), los individuos se verán afectados del mismo modo y se comportarán de una manera determinada (Hopcroft, 2016). Ahora bien, lo que sí podemos afirmar rotundamente es que estas enfermedades, entre otros factores, están generando modificaciones sociales en el comportamiento esperado por la colectividad y están ocasionando alteraciones de la morfogénesis social. Estos cambios todavía son demasiado leves, en lo relativo a las enfermedades poco frecuentes.

Pongamos algunos ejemplos. En algunos espectáculos culturales se está solicitando no aplaudir para evitar que los individuos que presentan una mayor sensibilidad al ruido puedan sentirse afectados negativamente. También se están introduciendo lentamente cambios en la señalización para facilitar la orientación y la percepción de ciudadanos con discapacidades visuales o intelectuales. Algunas personas comienzan a ser conscientes de que no pueden tocar, saludar o interactuar de la misma manera con todo el mundo, ya que es posible que tengan ante ellos a individuos afectados por algún tipo de hipersensibilidad o de alteración cutánea. En definitiva, existen determinantes biosociales que condicionan o modifican las interacciones sociales e, incluso, la forma en la que se está estructurando la sociedad.

Ahora bien, volvamos a las enfermedades no diagnosticadas. En este caso, la construcción social de la enfermedad es limitada, debido a que el enfermo no puede transmitir elementos que configuren los aspectos fundamentales que permitan estructurar la relación social. No obstante, siguen pesando sobre la enfermedad las significaciones culturales, de hecho, Susan Sontag afirmaba lo siguiente: "Basta ver una enfermedad cualquiera como un misterio, y temerla intensamente, para que se vuelva moralmente, si no literalmente, contagiosa" (Sontag 1996: 13). Esta construcción social del contagio, en relación con la enfermedad, es un elemento fundamental en la conformación de las relaciones sociales. Las personas sanas tienden a evitar el contacto con las que presentan alguna manifestación de carácter patológico (Coca, 2019b). Por otro lado, Conrad y Barker (2010) muestran la dificultad que tienen muchos enfermos para darle significado a sus enfermedades y reclamar un sentido de su propio self. Al fin y al cabo, las personas establecen, en sus relaciones sociales, su propia identidad (Ricoeur, 1996; Augé, 1996; Nair et al, 1999; Sartori, 2003). De ahí que la enfermedad se convierta en un fenómeno disruptivo de su realidad. Pues bien, imaginemos esto en una persona que sabe que está enferma, pero que no sabe lo que tiene. No existe una semiosis a la que podamos recurrir. El juego del lenguaje del que hablaba Wittgenstein (2009) parece no tener reglas. Al fin 
y al cabo, afirmó este filósofo, el lenguaje se asienta en el uso que hacemos de él y, por tanto, en las normas subyacentes. Ahora bien ¿qué se hace cuando no se muestra una realidad para designar? Dicho de otro modo, no existe un signo concreto que permita comprender rápidamente el significado y el significante de aquello designado. Podría haber un conjunto de signos vagos, cuyo significado llevara al mundo subjetivo del sufriente. La única opción a la que podría agarrarse es al uso de metáforas y analogías ¿Pero una metáfora de qué? El grave problema que se encuentra es precisamente éste: no hay referente, lo que supone que el código sígnico no puede ser institucionalizado y, por tanto, que existe una brecha biosemiótica y sociosemiótica de gran relevancia.

\section{El diagnóstico como utopía semiótica}

Hemos indicado previamente la existencia de brechas semióticas a nivel biológico (más concretamente, biomédico) y a nivel sociológico. Evidentemente el código biológico permanece (Barbieri, 2015 y 2019), pero la conexión de éste con el código sociológico es la que está afectada. Con el fin de romper con esta ausencia de signos para referirse a la realidad de las personas afectadas por enfermedades no diagnosticadas, la denominada comunidad científica está intentando transformar esta situación gracias al desarrollo de investigación básica y aplicada.

El principal proceso en el que se han puesto todas las esperanzas iniciales es en el diagnóstico de carácter tecnocientífico, es decir, basado en la la determinación genética de la enfermedad. Concretamente, para la determinación del problema genético causal, se están realizando varios enfoques posibles: el análisis parcial o completo del exoma. Recordemos que el exoma es el conjunto de genes codificantes de nuestro material genético, por lo que es en esta información codificable donde estará la causa de la enfermedad. Pues bien, el análisis parcial se realiza cuando más o menos se duda de la región donde podría encontrarse la alteración genética, cuando se sospecha la alteración de alguna proteína, cuando la persona tiene síntomas semejantes a los de otra patología conocida, etc. Es decir, que existe una información previa que permite dirigir el foco hacia zonas relativamente concretas del material genético. Así, el análisis completo del material genético codificable se suele realizar cuando se quiere rastrear todo el genoma codificable, normalmente debido a que no existe información previa al respecto. Las diferentes herramientas moleculares han permitido que en los últimos años se hayan descubierto una gran cantidad de enfermedades moleculares. Al respecto, téngase en cuenta que existen alrededor de 7.000 enfermedades raras (muchas de ellas descubiertas hace poco tiempo), mientras que se considera que hay unas 100 enfermedades comunes (Berman, 
2015). Por ello, las personas afectadas por enfermedades poco frecuentes y por las no diagnosticadas tienen su confianza puesta en este tipo de herramientas $\mathrm{y}$ en la posibilidad de encontrar un diagnóstico. Y es que este mecanismo de acción individual y colectiva permite una reducción social de la complejidad (Luhmann, 1996b) generada por la incertidumbre de una patología cuya causa se desconoce.

El mecanismo de confianza que opera en la mente de las personas ND no es deductivo, sino inductivo, según considera Luhmann (1996b). Al ver que otras personas han logrado obtener su diagnóstico a través de los mecanismos moleculares de diagnóstico, las personas ND confían que también lo conseguirán. Constituye, por tanto, un proceso utópico de confianza en el progreso de la ciencia. Los ND son, entonces, un sociotipo de personas en espera. Ahora bien, esta espera no es pasiva. Todo lo contrario. Los ND son conscientes de la confianza puesta en la razón (modernidad), aunque también saben que el sistema científico-tecnológico necesita ser dirigido y controlado. Por esta razón, apuestan por transformaciones en el sistema de salud que promuevan la aceleración de la materialización de esta utopía, de ese diagnóstico que todavía no tiene su lugar. Por ello, Gainotti et al, (2018) muestran que el establecimiento de procedimientos de diálogo entre los pacientes, las familias y los sanitarios está incrementando el éxito de estos diagnósticos tecnocientíficos.

Ahora bien, existe una situación de gran calado filosófico relacionado con estos diagnósticos tecnocientíficos a los que hemos hecho mención. Tal y como indica Wehling (2011), las personas del sociotipo ND, una vez que se determina el agente causal, pueden tardar en obtener una terapia efectiva, lo que los sitúa en un espacio imaginario entre la salud y la enfermedad. Al respecto, conviene tener en cuenta que el imaginario constituye el instrumento mismo de crítica de lo real (Ricoeur, 1988) y que puede representar una segunda vida, creada y superpuesta sobre la real (Lizcano, 2003). De ahí que la conformación del self siga planteando dificultades. En este sentido, Gayle Sulik (2009 y 2011) ha afirmado que se está produciendo lo que ella denomina como identidad tecnocientifica de la patología. En ella, la identidad está mediada por la información y las características biomédicas y no tanto por los elementos del mundo de la vida. En este sentido, Sulik (2009: 1062) advierte que las personas terminan identificándose de una manera muy fuerte con el agente causal e integran esta clasificación en su propio self. Este aspecto puede hacer olvidar, precisamente, el mundo de la vida e hipertrofiar el interés por el diagnóstico molecular. Evidentemente desconocemos las consecuencias de que esto se llevase a cabo, pero sabemos que no obviar los aspectos que nos hacen ser, precisamente, humanos ( $\mathrm{y}$, por tanto, el humanismo) no suele ser positivo a medio y largo plazo. 


\section{Conclusión}

Las enfermedades no diagnosticadas son uno de los grandes retos epistemológicos de la actualidad. En ellas se necesitan la conjugación de enfoques diferentes para intentar dar luz a la realidad humana generada por estas patologías. En este trabajo hemos mostrado como la ausencia de diagnóstico genera una brecha semiótica que necesita de puentes para que las personas no acaben estando situadas fuera de la estructura social. La historia nos ha mostrado que el conocimiento tiende a establecer categorías relativamente estancas, donde la idea de estandarización, tipologización o normalización juegan un papel fundamental. Este hecho, propio del desarrollo de la ciencia configura una estructura social basada en la pertenencia o no a aquello denominado como normal. De hecho, las distintas patologías son entendidas de esta manera. Pues bien, las enfermedades raras o poco frecuentes están transformando esta visión del mundo e incorporando la rareza como elemento estructurante de lo social. La discapacidad y los estudios sobre ella, ya habían dado pasos en este sentido. Es decir, en la incorporación de la diversidad como elemento configurador del gran sistema social. De ahí que el modo en que las personas nos relacionamos en sociedad va permitiendo, cada vez más, la diversidad en los comportamientos, en la apariencia, etc.

Las enfermedades no diagnosticadas todavía dan una vuelta de tuerca a esta dinámica de transformación social. Ello es debido a que las personas afectadas carecen de elementos denominadores de la realidad vivida. Esta compleja situación, en la que la persona afectada carece de elementos identificadores de su problema, sitúa en una zona "invisible" a estos sujetos. Ante esta situación, las emociones y vivencias se pueden sentir afectadas. Lo cual podría conducir a las personas afectadas a buscar denodadamente un elemento semiótico que les permita estructurar ese diálogo entre su realidad biomédica y la social. Actualmente este puente puede ser el diagnóstico molecular, aunque esta determinación no es exactamente un diagnóstico. Asimismo, este elemento semiótico también puede alterar la concepción de nuestro self y condicionar nuestras relaciones sociales y nuestra percepción del mundo de la vida. 


\section{Referencias bibliográficas}

Archer, M.S. (2009). Being Human: The Problem of Agency. Cambridge: Cambridge University Press.

Archer, M.S. (2010). Routine, Reflexivity, and Realism. Sociological Theory 28(3), 272-303. https://doi.org/10.1111/j.1467-9558.2010.01375.x

Archer, M.S. (2016). "Anormative Social Regulation: The Attempt to Cope with Social Morphogenesis". In: M.S. Archer (eds.) Morphogenesis and the Crisis of Normativity. Switzerland, Springer-Nature, pp. 141-168.

Augé, M. (1996). El sentido de los otros. Actualidad de la antropología. Barcelona: Paidós.

Balandier, G. (2014). El desorden. La teoría del caos y las ciencias sociales. Barcelona: GEDISA.

Barbieri, M. (2015). Code Biology. A New Science of Life. Dordrecht: Springer. Barbieri, M. (2019). Code Biology, Peircean Biosemiotics, and Rosen's Relational Biology. Biological Theory 14, 21-29.

Baudelaire, Ch. (2004). El pintor de la vida moderna. Murcia: Colegio Oficial de Aparejadores y arquitectos Técnicos, Consejería de Educación y Cultura y Fundación CAJAMURCIA.

Bauman, Z. (2003). Modernidad líquida. México: Fondo de Cultura Económica. Bauman, Z. (2004). La sociedad sitiada. Buenos Aires: F.C.E.

Baumer, F. L. (1985). El pensamiento europeo moderno. Continuidad y cambio en las ideas, 1600-1959. México: F.C.E.

Bellah, R. (2017). La religión en la evolución humana. Del Paleolítico a la era axial. Madrid: CIS.

Berman, J.J. (2015). Enfermedades raras y medicamentos huérfanos. Claves para entender y tratar las enfermedades comunes. Barcelona, Elsevier.

Berry, E. (2011). The role of the sociotype in managing chronic disease: Integrating bio-psycho-sociology with systems biology. Medical Hypotheses 77(4), 610-613.

Boudon, R. (1982). The Unintended Consequences of Social Action. London, Macmillan Press.

Coca, J.R. (2017). La Socio-hermenéutica Multidimensional como Teoría Social basada en los Imaginarios, la Irrealidad y la Utopía, Utopía y praxis latinoamericana 22 (76), 41-55.

Coca, J.R. (2019a). "Patologías de baja prevalencia en contexto social: Análisis teórico introductorio". In: Coca, J.R. (eds.) Enfermedades raras. Contribuciones a la investigación social y biomédica. Soria, CeasgaPublishing.

Coca, J.R. (2019b). (eds.) Enfermedades raras. Contribuciones a la investigación social y biomédica. Soria, Ceasga-Publishing. 
Coca, J.R., Rivas-Torres, A. y Cordero, A. (2020). Bases biológicas de la socialidad. Nova Acta Cientifica Compostelana (Bioloxía) 27: 51-58.

Coca, J.R., Sanz-Molina, L, Eraña, H. y Castilla, J. (2019). Análisis cualitativo del impacto social y familiar de las encefalopatías espongiformes transmisibles humanas. Revista de Neurología 69(6), 242-249. https://doi. org/10.33588/rn.6906.2019122

Conrad, P. y Barker, K.K. (2010). The Social Construction of Illness. Key Insights and Policy Implications. Journal of Health and Social Behavior 51(S): S67-S79. https://doi.org/10.1177/0022146510383495

Elias, N. (2000). Teoría del símbolo. Un ensayo de antropología cultural. Barcelona: Península.

Endress, M. (2014). "Interpretive Sociologies and Traditions of Hermeneutics". In: Staudigl M., Berguno G. (eds) Schutzian Phenomenology and Hermeneutic Traditions. Contributions to Phenomenology, vol 68. Springer, Dordrecht. 33-54. https://doi.org/10.1007/978-94-007-6034-9_3

Farson, R.E. (1973). Las potencialidades humanas, en VVAA, Ensayos sobre el Apocalipsis. Barcelona: Kairós.

Flyvbjerg, B. (2001). Making Social Science Matter. Why social inquiry fails and how it can succeed again. Cambridge: Cambridge University Press.

Gadamer, H.G. (1984). Verdad y método. Fundamentos de una hermenéutica filosófica. Salamanca: Sígueme.

Gainotti, S., Mascalzoni, D., Bros-Facer, V., Petrini, C., Floridia, G., Roos, M., Salvatore, M. y Taruscio, D. (2018). "Meeting Patients' Right to the Correct Diagnosis: Ongoing International Initiatives on Undiagnosed Rare Diseases and Ethical and Social Issues". International Journal of Environmental Research and Public Health 15, 2072. https://doi.org/10.3390/ijerph15102072

Gaspar, S. (2003). Consecuencias no intencionales y figuración: una incursión crítica en la obra de Norbert Elias. Reis, 119-148.

Giddens, A. (2013). The Constitution of Society: Outline of the Theory of Structuration. New York: John Wiley \& Sons.

Ginev, D. (2016). Hermeneutic Realism. Reality Within Scientific Inquiry. Switzerland: Springer-Nature.

Goffman, E. (2009). La presentación de la persona en la vida cotidiana. Buenos Aires: Amorrortu.

González, J.M. (2006). La diosa Fortuna. Metamorfosis de una metáfora política. Madrid: Antonio Machado libros.

Grondin, J. (2003). Introducción a Gadamer. Barcelona: Herder.

Heidegger, M. (1971). El ser y el tiempo. México: Fondo de Cultura Económica. Hopcroft, R.L. (2016). Grand Challenges in Evolutionary Sociology and Biosociology. Frontiers in Sociology 1, article 2. https://doi.org/10.3389/ fsoc. 2016.00002 
Jaspers, K. (2017). Origen y meta de la historia. Barcelona: Acantilado. Koselleck, R. (2016). historia/Historia. Madrid: Trotta.

Kuper, H., Banks, L. M., Bright, T., Davey, C., y Shakespeare, T. (2020). Disability-inclusive COVID-19 response: What it is, why it is important and what we can learn from the United Kingdom's response. Wellcome open research, $5,79$.

Lamo de Espinosa, E. (1990). La sociedad reflexiva. Madrid: Siglo XXI.

Lizcano E. (2003). "Imaginario colectivo y análisis metafórico", transcripción de la conferencia inaugural del Primer Congreso Internacional de Estudios sobre Imaginario y Horizontes Culturales que se celebró en la Universidad Autónoma del Estado de Morelos, Cuernavaca, México, del 6 al 9 de mayo. http://www.unavarra.es/puresoc/pdfs/c salaconfe/SC-Lizcano-2.pdf

Luhmann, N. y De Giorgi (1993). Teoría de la sociedad. México: Universidad de Guadalajara / Universidad Iberoamericana.

Luhmann, N. (1996a). "La contingencia como atributo de la sociedad moderna", en Las consecuencias perversas de la modernidad: modernidad, contingencia y riesgo. Barcelona: Anthropos.

Luhmann, N. (1996b). Confianza. Barcelona: Anthropos.

Maffesoli, M. (2020). "De L'Universalisme à 1'uni-diversité postmoderne", en Celso Sánchez-Capdequí y Juan A. Roche Cárcel, Modernidades regresivas. Madrid: CIS (en prensa).

Marijuán P.C., Montero-Marín, J., Navarro, J., García-Campayo, J. y del Moral, R. (2017). The "sociotype" construct: Gauging the structure and dynamics of human sociality. PLOS ONE 12(12), e0189568. https://doi.org/10.1371/ journal.pone.0189568

Merton, R. (1936). The Unanticipated Consequences of Purposive Social Action. American Sociological Review, 1(6), 894-904.

Moulines, C.U. (2011). El desarrollo moderno de la filosofía de la ciencia (1890-2000). México: UNAM/IIF.

Nair, S., De Jucas, J. (1999). Inmigrantes. El desplazamiento en el mundo. Madrid: Ministerio de Trabajo y Asuntos Sociales. Secretaría General de Asuntos Sociales. Instituto de Migraciones y Servicios Sociales.

Nescolarde-Selva, J.A., Gash, H. y Usó-Domenech, J.L. (2019). What are unintended and adverse consequences?, Kybernetes 48(2), 226-237. https://doi.org/10.1108/K-12-2017-0466

Ortega, M., Gómez-Chaparro, J.L., González-Meneses, A., Guillén, J., Varo, A. y Fernández, E. (2012). Mapas conceptuales para el diagnóstico de enfermedades raras en atención primaria. Atención primaria 44(1), 43-50. https://dx.doi.org/10.1016\%2Fj.aprim.2011.01.009

Palau, F. (2020). Enfermedades raras. Ciencia y realidad de la rareza en medicina. Madrid: Los libros de la catarata. 
Picó, J. (1988). “Introducción”, Modernidad y Posmodernidad. Madrid: Alianza.

Ramos, R. (1993). "Una aproximación a las paradojas de la acción social". Problemas de teoría social contemporánea. Madrid: CIS.

Reed, I.A. (2011). Interpretation and Social Knowledge. On the Use of Theory in the Human Sciences. Chicago: The University of Chicago Press.

Reichertz, J. (1988). Verstehende Soziologie ohne Subjekt? Die objektive Hermeneutik als Metaphysik der Strukturen. Kölner Zeitschrift für Soziologie und Sozialpsychologie 40, 207-222.

Ricoeur, P. (1988). Hermenéutica y Acción. De la Hermenéutica del Texto a la Hermenéutica de la Acción. Buenos Aires: Prometeo Libros-UCA.

Ricoeur, P. (1996). Sí mismo como otro. España: Siglo XXI.

Roche, J.A. (2009). La sociedad evanescente. Barcelona: Anthropos.

Sabatello, M., Blankmeyer, T., McDonald, K.E. y Appelbaum, P.S. (2020). Disability, Ethics, and Health Care in the COVID-19 Pandemic. American Journal of Public Health 110, 1523-1527.

Sánchez Capdequí, C. (2004). Las máscaras del dinero. El simbolismo social de la riqueza. Barcelona: Anthropos-Universidad Autónoma Metropolitana.

Sartori, Giovanni (2003). La sociedad multiétnica. Pluralismo, multiculturalismo, extranjeros e islámicos. Madrid: Taurus.

Schutz, A. (1967). The phenomenology of the social world. Evanston, Northwestern University Press.

Sontag, S. (1996). La enfermedad y sus metáforas y El sida y sus metáforas. Madrid: Taurus.

Sulik, G. (2009). Managing Biomedical Uncertainty: The Technoscientific Illness Identity. Sociology of Health \& Illness 31(7), 1059-76. DOI: https://doi.org/10.1111/j.1467-9566.2009.01183.x

Sulik, G.A. (2011). 'Our Diagnoses, Our Selves': The Rise of the Technoscientific Illness Identity. Sociology Compass 5 (6), 463-477. DOI: https://doi.org/10.1111/j.1751-9020.2011.00374.X

Taruscio, D., Groft, S. C., Cederroth, H., Melegh, B., Lasko, P., Kosaki, K., Baynam, G., McCray, A. y Gahl, W.A. (2015). Undiagnosed Diseases Network International (UDNI): White paper for global actions to meet patient needs. Molecular genetics and metabolism, 116(4), 223-225. https://doi.org/10.1016/j.ymgme.2015.11.003

Thiebaut, C. (1996). "La mal llamada postmodernidad (o las contradanzas de lo moderno)", en Valeriano Bozal, Historia de las ideas estéticas y de las teorías artísticas contemporáneas, Vol. II. Madrid: Visor.

Tifft, C.J. y Adams, D.R. (2014). The National Institutes of Health undiagnosed diseases program. Current Opinion in Pediatrics 26(6), 626-633. 
Tuomela, R. (2010). The Philosophy of Sociality. The Shared Point of View. Oxford: Oxford University Press.

Tuomela, R. (2013). Social Ontology. Collective Intentionality and Group Agents. Oxford: Oxford University Press.

Veblen, Th. (2008). Teoría de la clase ociosa. Madrid: Alianza.

Ward, L.F. (1905). Evolution of social structures. American Journal of Sociology 10(5), 589-605.

Ward, L.F. (1921). Pure sociology. On the origin and spontaneous development of society. London: Macmillan Company.

Wehling, P. (2011). The "technoscientization" of medicine and its limits: technoscientific identities, biosocialities, and rare disease patient organizations. Poiesis \& Praxis 8 (2-3), 67-82. DOI: http://doi.org/10.1007/s10202-0110100-3

Wittgenstein, L (2009). Wittgenstein I. Madrid: Gredos.

Zubiri, X. (1998). Sobre el hombre. Madrid: Alianza. 
